\title{
Lifting the mask on neurological manifestations of COVID-19
}

\section{Alessandro Pezzini(D) and Alessandro Padovani}

Abstract | As the severe acute respiratory syndrome coronavirus 2 (SARS-CoV-2) epidemic spreads, it is becoming increasingly evident that coronavirus disease 2019 (COVID-19) is not limited to the respiratory system, and that other organs can be affected. In particular, virus-related neurological manifestations are being reported more and more frequently in the scientific literature. In this article, we review the literature on the association between COVID-19 and neurological manifestations, present evidence from preclinical research suggesting that SARS-CoV-2 could be responsible for many of these manifestations, and summarize the biological pathways that could underlie each neurological symptom. Understanding the mechanisms that lead to neurological manifestations in patients with COVID-19 and how these manifestations correlate with clinical outcomes will be instrumental in guiding the optimal use of targeted therapeutic strategies.

After the first cases of the novel coronavirus disease 2019 (COVID-19) were reported in Wuhan, China, in December 2019, the spread of severe acute respiratory syndrome coronavirus 2 (SARS-CoV-2) rapidly became a pandemic ${ }^{1}$, forcing health-care systems and governments across the world to take extreme measures to contain the infection, and simultaneously engaging the scientific community in a race against time to develop effective treatments. Although evidence indicates that SARS-CoV-2 can be aerosolized or detected in the stool, the virus is spread predominantly via respiratory droplets from both symptomatic and asymptomatic infected individuals ${ }^{2,3}$. As with many other viral syndromes, the most common symptoms of COVID-19 are fever and dry cough, whereas other manifestations, including rhinorrhoea and gastrointestinal symptoms, are much less frequent ${ }^{3}$. Reports from China at the beginning of the outbreak and from other countries afterwards, have clearly demonstrated that most patients (81\%) have mild symptoms with no pneumonia or mild pneumonia, and, among those patients with more significant symptoms, $14 \%$ have severe respiratory distress and $5 \%$ have respiratory failure, septic shock, and/or multi-organ failure ${ }^{4}$.

Although we are still in the early phases of our attempts to understand the syndromic complexity of COVID-19, growing evidence indicates that the disease is not limited to the respiratory system and that SARS-CoV-2 has an organotropism beyond the respiratory tract, including the kidneys, liver, heart, skin and brain. In particular, as the disease spreads, neurological manifestations in patients with COVID-19 are reported more and more frequently in the scientific literature ${ }^{5,6}$. In this article, we provide evidence to support the hypothesis that the SARS-CoV-2 might be responsible for neurological symptoms, discuss potential underlying biological mechanisms, and pose new research questions.

\section{Epidemiological data}

The literature regarding neurological features of COVID-19 comprises a large number of case reports and only a few case series (TABLE 1). Therefore, correctly estimating incidence and prevalence is difficult. Unavoidable methodological differences among the studies also present a challenge to the interpretation of the results as a whole.

In the first case series from Wuhan, China, Mao and colleagues detected neurological symptoms in 78 out of 214 patients (36.4\%) with COVID-19 (REF.'), and an analysis of the ALBACOVID registry in Spain by Romero-Sánchez and colleages ${ }^{8}$ detected neurological symptoms in 483 out of 841 patients (57.4\%) with COVID-19. Pinna and colleagues detected neurological symptoms in 50 out of 650 patients $(7.7 \%)$ hospitalized with COVID-19 in Chicago, Illinois ${ }^{9}$, and Karadaş and colleagues detected neurological symptoms in 83 out of 239 patients (34.7\%) with COVID-19 in Ankara, Turkey ${ }^{10}$. In a case series from 56 hospitals designated as COVID-19 treatment centres in China, Xiong and colleagues detected new-onset specific neurological events in 39 out of 917 patients (4.2\%) with COVID-19 (REF. ${ }^{11}$ ).

Although these analyses provide useful information on the potential neurological manifestations related to the infection, several inherent limitations should be considered when interpreting the results. First, the category 'neurological symptoms' is vague and poorly defined and this can lead to variability between studies. For example, in contrast to other studies, Xiong and colleagues excluded from the group of patients with neurological manifestations those who only had non-specific symptoms, such as headache, dizziness, fatigue and myalgia, which are all likely to be caused by the systemic condition, as well as patients whose neurological symptoms, such as impaired consciousness, could be fully accounted for by sedation during ventilation ${ }^{11}$. Second, as the data used in most of these studies were extracted from electronic medical records, some neurological symptoms might not have been captured by the analyses, for example mild symptoms, such as taste and smell impairment, or neurological symptoms in patients with very severe disease. Furthermore, during the outbreak, advanced neuroimaging, such as MRI, and diagnostic procedures, such as lumbar puncture and electromyography, were purposely avoided to reduce the risk of cross-infection. Therefore, in all these studies, most of the recorded symptoms were based on subjective descriptions provided by the patient. Finally, as these studies were hospital based, they do not necessarily reflect the true incidence of neurological manifestations in individuals with SARS-CoV-2 infection in the community.

Three other series of patients with COVID-19 in whom neurological 
Table 1 | Incidence of neurological manifestations associated with COVID-19

\begin{tabular}{|c|c|c|c|c|c|c|c|c|c|}
\hline Clinical feature or diagnosis & $\begin{array}{l}\text { Mao } \\
\text { et al. }{ }^{7}\end{array}$ & $\begin{array}{l}\text { Romero-Sánchez } \\
\text { et al. }{ }^{8}\end{array}$ & $\begin{array}{l}\text { Pinna } \\
\text { et al. }{ }^{9}\end{array}$ & $\begin{array}{l}\text { Karadaş } \\
\text { et al. }{ }^{10}\end{array}$ & $\begin{array}{l}\text { Xiong } \\
\text { et al. }{ }^{11}\end{array}$ & $\begin{array}{l}\text { Helms } \\
\text { et al. }{ }^{12}\end{array}$ & $\begin{array}{l}\text { Benussi } \\
\text { et al. }{ }^{13}\end{array}$ & $\begin{array}{l}\text { Paterson } \\
\text { et al. }{ }^{14}\end{array}$ & $\begin{array}{l}\text { Chen } \\
\text { et al. } .^{15}\end{array}$ \\
\hline COVID-19 (total number of patients) & 214 & 841 & 650 & 239 & 917 & 58 & 56 & 43 & 274 \\
\hline $\begin{array}{l}\text { COVID-19 with neurological } \\
\text { manifestations (number (\%) of patients) }\end{array}$ & $78(36.4)$ & $483(57.4)$ & $50(7.7)$ & $83(34.7)$ & $39(4.2)$ & 49 (84.4) & $56(100)^{b}$ & $43(100)^{b}$ & $78(28.4)$ \\
\hline \multicolumn{10}{|l|}{ CNS manifestations ${ }^{a}$} \\
\hline Overall & $53(67.9)$ & NR & NR & NR & NR & NR & NR & $35(81.4)$ & NR \\
\hline Dizziness & $36(46.1)$ & $51(10.5)$ & NR & $16(19.2)$ & NR & NR & NR & NR & $21(7.6)$ \\
\hline Headache & $28(35.9)$ & $119(24.6)$ & $12(24)$ & $64(77.1)$ & $2(5.1)$ & NR & NR & NR & $31(11.3)$ \\
\hline Impaired consciousness & $16(20.5)$ & $165(34.1)$ & $30(60)$ & $23(27.7)$ & $25(64.1)$ & NR & NR & $7(16.2)$ & $26(9.5)$ \\
\hline Acute stroke & $6(7.7)$ & $14(2.9)$ & $20(40)$ & $9(10.8)$ & $10(25.6)$ & NR & $43(76.8)$ & $8(18.6)$ & NR \\
\hline Ataxia & $1(1.3)$ & NR & $1(2)$ & NR & NR & NR & NR & NR & NR \\
\hline Seizures & $1(1.3)$ & $6(1.2)$ & $13(26)$ & NR & $0(0.0)$ & NR & $4(7.1)$ & NR & NR \\
\hline Agitation & NR & NR & NR & NR & NR & $40(6.9)$ & NR & NR & NR \\
\hline Confusion & NR & $69(14.2)$ & NR & NR & NR & $26(65.0)$ & NR & $10(23.2)$ & NR \\
\hline Corticospinal tract signs & NR & NR & NR & NR & NR & $39(67.2)$ & NR & $5(11.6)$ & NR \\
\hline Dysexecutive syndrome & NR & NR & NR & NR & NR & $14(35.8)$ & NR & NR & NR \\
\hline Other & NR & NR & NR & NR & NR & NR & $9(16.1)$ & $3(6.9)$ & NR \\
\hline Neuropsychiatric symptoms & NR & $167(34.5)$ & NR & NR & NR & NR & NR & NR & NR \\
\hline Movement disorders & NR & $6(1.2)$ & NR & NR & $2(5.1)$ & NR & NR & NR & NR \\
\hline Encephalitis & NR & $1(0.2)$ & NR & NR & $0(0.0)$ & NR & NR & $12(27.9)$ & NR \\
\hline \multicolumn{10}{|l|}{ PNS manifestations ${ }^{a}$} \\
\hline Overall & $19(24.3)$ & NR & NR & $53(22.1)$ & NR & NR & NR & $8(18.6)$ & NR \\
\hline Anosmia & $11(14.1)$ & $41(8.5)$ & $3(6)$ & $18(21.7)$ & NR & NR & NR & NR & NR \\
\hline Dysgeusia & $12(15.4)$ & $52(10.7)$ & $5(10)$ & $16(19.2)$ & NR & NR & NR & NR & NR \\
\hline Dysautonomia & NR & $21(4.3)$ & $6(12)$ & NR & NR & NR & NR & NR & NR \\
\hline AIDP & NR & $1(0.2)$ & $0(0)$ & $1(1.2)$ & NR & NR & NR & $7(16.2)$ & NR \\
\hline \multicolumn{10}{|l|}{ Skeletal muscle manifestations ${ }^{a}$} \\
\hline Overall & $23(29.5)$ & $253(52.3)$ & $6(12)$ & $36(43.3)$ & $2(5.1)$ & NR & NR & NR & NR \\
\hline
\end{tabular}

AIDP, acute inflammatory demyelinating polyneuropathy; COVID-19, coronavirus disease 2019; NR, not reported. ${ }^{a}$ Number (percentage) of patients with neurological symptoms. bStudies included only patients with neurological manifestations.

manifestations were systematically assessed have peculiar characteristics that make them not fully comparable to the studies mentioned above. In particular, Helms and colleagues investigated a selected series of 58 patients consecutively admitted to two intensive care units in Strasbourg, France, because of acute respiratory distress syndrome (ARDS) due to COVID-19 (REF. ${ }^{12}$ ). The severity of the disease in these patients is likely to have prevented clinicians from performing an accurate neurological evaluation. The 56 patients with COVID-19 included in a study by Benussi and colleagues were hospitalized in Brescia, Italy, because of neurological diseases ${ }^{13}$, which would obviously have prevented any assessment of the frequency of neurological manifestations in those with confirmed infection. The same is true for the 43 patients with COVID-19 in the UK reported by Paterson and colleagues ${ }^{14}$. A further series of patients with COVID-19 reported by Chen and colleagues ${ }^{15}$ provides sparse information on several neurological features; however, a systematic search for symptoms resulting from the involvement of the nervous system was not performed. These small series do not reflect the entire spectrum of neurological features in COVID-19 disease and much is still to be learned, and this is an issue that can only be addressed by thorough neurological testing in large cohorts of patients with confirmed infection. However, the overall picture that emerges from these reports is characterized by neurological manifestations that range from fairly non-specific symptoms, for example, myopathy, dizziness or headache, to more specific symptoms, for example, anosmia and ageusia, impaired consciousness, seizures or stroke. Interestingly, although some of the common non-specific symptoms tend to manifest early in the clinical course of the disease, most of the specific symptoms, with the exception of anosmia and ageusia, are reported in severely affected individuals.

\section{Underlying biological mechanisms}

The epidemiological findings discussed above raise the crucial question of whether and, if so, to what extent these neurological symptoms are caused by SARS-CoV-2 as opposed to being general complications of critical illness. If SARS-CoV-2 is responsible for these symptoms, a second important question to answer is whether such neurological features are attributable to direct damage to the nervous system by the virus or to secondary, parainfectious, mechanisms. Given the high rates of SARS-CoV-2 infection in the general population ${ }^{1}$, coincidental occurrence of neurological diseases is likely. Moreover, most of the neurological symptoms found in 
the studies mentioned above are common in many severe infections. Even anosmia and ageusia, which have received special attention during the COVID-19 outbreak, are ubiquitous in other common upper respiratory tract infections. Therefore, we must be cautious about inferring any specific causal links between SARS-CoV-2 and neurological symptoms.

\section{Evidence from other coronaviruses.}

SARS-CoV-2 is a novel single-stranded enveloped RNA virus and the seventh known human coronavirus. This novel virus is unlike the coronaviruses that cause the common cold, that is, 229E, OC43, NL63 and HKU1, but is similar to the severe acute respiratory syndrome coronavirus (SARS-CoV) ${ }^{16}$ and the Middle East respiratory syndrome coronavirus $(\mathrm{MERS}-\mathrm{CoV})^{17}$. Like many other coronaviruses, SARS-CoV-2 is believed to have originated in bats, as it shares $79.5 \%$ genome sequence identity with SARS-CoV and $89-96 \%$ nucleotide identity with bat coronaviruses $^{18}$. SARS-CoV-2 is assumed to have moved from bats to an intermediate host, possibly a Malayan pangolin, and then, like SARS-CoV and MERS-CoV, to humans ${ }^{19}$.

Of the identified coronaviruses, at least three - 229E, OC43 (REFS ${ }^{20-23}$ ) and SARS-CoV ${ }^{24,25}$ - are neuroinvasive and neurotropic. Both SARS-CoV-2 and SARS-CoV enter human cells via the angiotensin-converting enzyme 2 (ACE2) receptor ${ }^{26}$, which is an important component of the renin-angiotensin system in the brain ${ }^{27}$. Therefore, like SARS-CoV, SARS-CoV-2 might also be able to invade the CNS. In addition, the structure and mode of replication of SARS-CoV-2 are similar to those of neuroinvasive animal coronaviruses ${ }^{28}$, such as porcine haemagglutinating encephalitis virus ${ }^{29}$, feline coronavirus ${ }^{30}$ and mouse hepatitis virus $(\mathrm{MHV})^{31}$. MHV, in particular, has been shown to persist in the mouse CNS after acute infection and to induce an immune-mediated, chronic demyelinating disease, similar to multiple sclerosis in humans $s^{32,33}$. Taken together, these observations have led to speculation about the possible involvement of SARS-CoV-2 in neurological diseases; however, definitive conclusions about neuroinvasivity cannot yet be drawn, as the mechanism by which the virus might reach the human CNS remains poorly defined, and definitive and specific associations of SARS-CoV-2 with any known human neuropathology are missing.
Neuroinvasivity of SARS-CoV-2. An important sign that SARS-CoV-2 can invade the CNS would be the detection of the virus in cerebrospinal fluid (CSF). In May 2020, Moriguchi and colleagues ${ }^{34}$ were the first to report the presence of SARS-CoV-2 RNA, detected using real-time reverse transcription PCR (RT-PCR) in the CSF of a patient with encephalopathy and COVID-19, and further cases have since been reported ${ }^{35-38}$. However, SARS-CoV-2 was not detected in the CSF of the majority of patients with neurological manifestations and proven SARS-CoV-2 infection in whom RT-PCR was performed ${ }^{12,39-41}$. In addition, PCR techniques can be at risk of sample contamination, which can lead to false-positive results, although this issue is unlikely to be a concern in experienced laboratories ${ }^{42}$.

At least theoretically, this failure to detect SARS-CoV-2 in the CSF of the majority of individuals with encephalopathy and COVID-19 could have several explanations. First, the virus could be mainly cell-bound and spread from cell to cell without entering the CSF. Second, the virus might have been present in the CSF at concentrations below the level of detection of the testing method because of a delay in CSF sampling. Third, the presence of haem products resulting from the breakdown of erythrocytes in the CSF could inhibit the action of the polymerase and, thus, the detection of SARS-CoV-2. Alternatively, the failure to detect SARS-CoV-2 in CSF despite evidence of inflammation, including CSF pleocytosis and elevated CSF protein levels, raises the possibility that in some individuals COVID-19-related encephalitis might result from immune-mediated inflammatory mechanisms, in the absence of direct virus invasion.

Regarding the detection of SARS-CoV-2 in CNS tissue samples, Paniz-Mondolfi and colleagues provided the first evidence of SARS-CoV-2 viral particles in the cytoplasm of frontal lobe neurons, as well as in brain endothelial cells, in the post-mortem examination of a patient with COVID-19 who presented with confusion and encephalopathy during the course of the infection ${ }^{40}$. More recently, data from an autopsy series of 27 patients who died from COVID-19 indicated that, although SARS-CoV-2 preferentially infects cells in the respiratory tract, it can be detected in multiple organs, including the brain, albeit with lower viral load ${ }^{43}$. The detection of viral RNA in human brain samples would support the hypothesis that SARS-CoV-2 is naturally neuroinvasive and that it might establish a persistent infection in the CNS in humans. However, these results need to be confirmed, and some notable data are available which suggest that the virus is not detectable in human brain tissue $\mathrm{e}^{41,44}$.

Possible route of neuroinvasion. Viruses can enter the CNS through two distinct routes: haematogenous dissemination and neuronal retrograde dissemination. In haematogenous dissemination the virus spreads throughout the body via the bloodstream and then enters the brain by crossing the bloodbrain barrier, whereas retrograde viral dissemination towards the CNS occurs when a virus infects neurons in the periphery and uses the transport machinery within those cells to gain access to the $\mathrm{CNS}^{45}$.

Although the neuroinvasivity of SARS-CoV-2 has not yet been confirmed, multiple lines of evidence suggest that other human coronaviruses can use both the haematogenous route and neuronal dissemination to penetrate the $\mathrm{CNS}^{46-48}$. After infecting the airways, coronaviruses can, under certain circumstances, pass through the epithelial barrier, gain access to the bloodstream and then enter the CNS by either infecting endothelial cells of the blood-brain barrier or epithelial cells of the blood-CSF barrier in the choroid plexus. Furthermore, evidence suggests that coronaviruses can infect leukocytes, that is, lymphocytes, granulocytes, monocytes and monocyte derivatives. Once activated by infection, these leukocytes disseminate towards other tissues, and cross the blood-brain barrier to access the CNS. This process has been referred to as a Trojan horse mechanism ${ }^{47}$. In the CNS, leukocytes produce pro-inflammatory cytokines such as TNF that can damage oligodendrocytes and/or neurons, and chemokines such as CCL5, CXCL10 and CXCL11 that induce chemoattraction of activated $\mathrm{T}$ cells and/or other leukocytes ${ }^{47}$. After sensing infection, astrocytes can also produce chemokines, including CCL2, CCL5 and CXCL12, that participate in the recruitment of more infected leukocytes. SARS-CoV-2 might, therefore, initiate an aberrant neuroinflammatory loop, which results in neuropathology ${ }^{48}$. Alternatively, coronaviruses have been shown to infect vascular endothelial cells, which then spread the virus directly to glial cells in the $\mathrm{CNS}^{27,47,48}$.

SARS-CoV-2 might also reach the CNS via neuronal dissemination. A growing body of evidence indicates that many human and non-human viruses, including herpes simplex virus, human immunodeficiency 
virus and several coronaviruses ${ }^{45,49}$, invade peripheral nerve terminals, spread retrogradely across nerve synapses, and thus gain access to the CNS. In particular, following intranasal infection, some viruses infect the olfactory receptor neurons, pass through the neuroepithelium of the olfactory mucosa to reach the olfactory bulb, gain access to the mitral cells and the olfactory nerve, and from there eventually spread to the hippocampus and other brain structures ${ }^{44,50}$. The possibility that SARS-CoV-2 can also enter the CNS via this olfactory route has received considerable attention in recent months ${ }^{51}$.

At the cellular level, the binding of the spike protein on the surface of SARS-CoV-2 to the ACE2 receptor on the host cell is central to infection ${ }^{26}$, as it is for the cell entry of two other coronaviruses SARS-CoV and NL63 (REF. ${ }^{52}$ ). Spike proteins are crown-shaped membrane fusion proteins distributed across the surface of all coronaviruses. Although SARS-CoV and SARS-CoV-2 share similar spike protein and ACE2 receptor-binding sequences, the affinity of the SARS-CoV-2 spike protein for the ACE2 receptor seems to be $10-20$-fold higher than that of the SARS-CoV spike protein, suggesting that SARS-CoV-2 has a much higher human-to-human transmissibility than SARS-CoV ${ }^{53,54}$. Following binding to the ACE2 receptor, the transmembrane protease serine 2 (TMPRSS2) ${ }^{55}$ induces proteolytic cleavage and 'priming' of the spike protein. Once primed, the spike protein can induce virus entry into the cell. Although evidence suggests that spike protein priming is also partly dependent on the endosomal cysteine proteases cathepsin B (CatB) and cathepsin L $(\mathrm{CatL})^{56}$, it is TMPRSS2, not CatB and CatL, that is essential for viral entry into primary target cells and for viral spread in the infected host ${ }^{57,58}$. Therefore, ACE2 receptors are crucial for SARS-CoV-2 cellular tropism in humans. In humans, the ACE2 receptor is expressed in airway epithelia, kidney cells, small intestine, lung parenchyma, vascular endothelium and widely throughout the $\mathrm{CNS}^{4}$. Information about the localization of ACE2 receptor expression within the human brain is emerging. Recent reports indicate that ACE2 is expressed in neurons, astrocytes and oligodendrocytes, as well as in the substantia nigra, ventricles, middle temporal gyrus, posterior cingulate cortex and olfactory bulb ${ }^{59}$. Such widespread expression of the ACE2 receptor in the brain has led to speculation that, like SARS-CoV, SARS-CoV-2 has the potential to infect neurons and glial cells throughout the CNS.
Neuropathology. Although gaps in our understanding of the neuropathology underlying the observed neurological features remain, SARS-CoV-2-associated neuropathology is gradually being uncovered. In May 2020, Reichard and colleagues described several types of pathological lesions that might contribute to the neurological manifestations observed in patients with COVID-19 $\left(\mathrm{REF}^{60}{ }^{6}\right.$. Post-mortem examination of a 71-year-old patient with confirmed SARS-CoV-2 infection revealed a range of neuropathological lesions resembling both vascular and demyelinating aetiologies. These lesions included haemorrhagic white matter lesions, which are a characteristic feature of acute haemorrhagic necrotizing encephalitis (ANE), with surrounding axonal injury and macrophages. The subcortical white matter had clusters of macrophages, myelin loss and a perivascular acute disseminated encephalomyelitis (ADEM)-like appearance. Focal microscopic areas of necrosis with central loss of white matter and marked axonal injury, and rare neocortical microscopic infarcts were also identified. Overall, the lack of the typical features of viral and post-viral encephalitides in this patient argues against the hypothesis of a direct damaging effect of the virus on the CNS and, indirectly, prompts speculation that, after a latent period following the infectious illness, SARS-CoV-2 might induce a secondary, parainfectious process that is responsible for many neurological manifestations.

In the few other neuropathological studies currently available non-specific findings or no abnormalities at all were detected $\mathrm{d}^{40,41,43,44,61}$ (TABLE 2). This heterogeneity is probably influenced by the fact that, although the patients with COVID-19 who underwent neuropathological analysis all showed signs of CNS involvement, they had different neurological features and probably different neurological diseases.

Neuroimaging. Neuroimaging data can also help us understand the effect of SARS-CoV-2 on the CNS. Unfortunately, published brain imaging findings from patients with confirmed COVID-19 are currently scarce and limited to small case series. In this section, we discuss some of these initial findings. In June 2020, Nicholson and colleagues performed brain magnetic resonance susceptibilityweighted imaging (SWI) in a series of four patients with COVID-19 and observed a multifocal abnormal signal, potentially with both arterial and venous components ${ }^{62}$.
The authors interpreted these findings, together with evidence of multifocal cortical infarcts in some of the patients, as a marker of arterial microvascular thrombosis, whereas the hyperdense veins detected in other patients were considered to be indicative of sluggish venous flow. Furthermore, in a subset of patients, the authors detected multifocal petechial bleeding and eventual massive haemorrhage, which they considered to be the end result of diffuse thrombosis leading to blood-brain barrier breakdown.

Soon after Nicholson et al. reported their findings, a study by Coolen and colleagues found macrohaemorrhagic and microhaemorrhagic lesions as well as posterior reversible encephalopathy syndrome (PRES)-related brain lesions in a small series of post-mortem brain MRI scans from patients who had died with COVID-19 $\left(\right.$ REF. $\left.^{63}\right)$. These findings indirectly support the hypothesis of a prominent role of microangiopathy and microthrombosis, as well as of blood-brain barrier dysfunction in the pathogenesis of cerebrovascular manifestations in patients with COVID-19. In addition, Kremer and colleagues observed three main neuroradiological patterns in a series of 37 patients with severe COVID-19: signal abnormalities located in the medial temporal lobe; non-confluent multifocal white matter hyperintense lesions with variable enhancement on fluid-attenuated inversion recovery (FLAIR) and diffusion sequences associated with haemorrhagic lesions; and extensive and isolated white matter microhaemorrhages ${ }^{38}$. In a series of 11 critically ill patients with COVID-19, Radmanesh and colleagues observed confluent $\mathrm{T} 2$ hyperintensity and mild restricted diffusion in bilateral supratentorial deep and subcortical white matter, in combination with multiple punctate microhaemorrhages in juxtacortical and callosal white matter ${ }^{64}$. As Radmanesh and colleagues concluded, these lesions were likely to have been complications of hypoxaemia, although other potential aetiologies, such as direct cerebral infection by SARS-CoV-2, sepsis-associated encephalopathy, post-infectious demyelinating or haemorrhagic encephalitis, toxic and metabolic aetiologies, and PRES cannot be excluded. Although the underlying mechanism of brain abnormalities detected on MRI remains unsolved, and a direct role of SARS-CoV-2 is far from clear, these findings provide further evidence that CNS damage can occur in patients with COVID-19. 
Table 2 | Findings from neuropathological studies of patients with SARS-CoV-2 infection

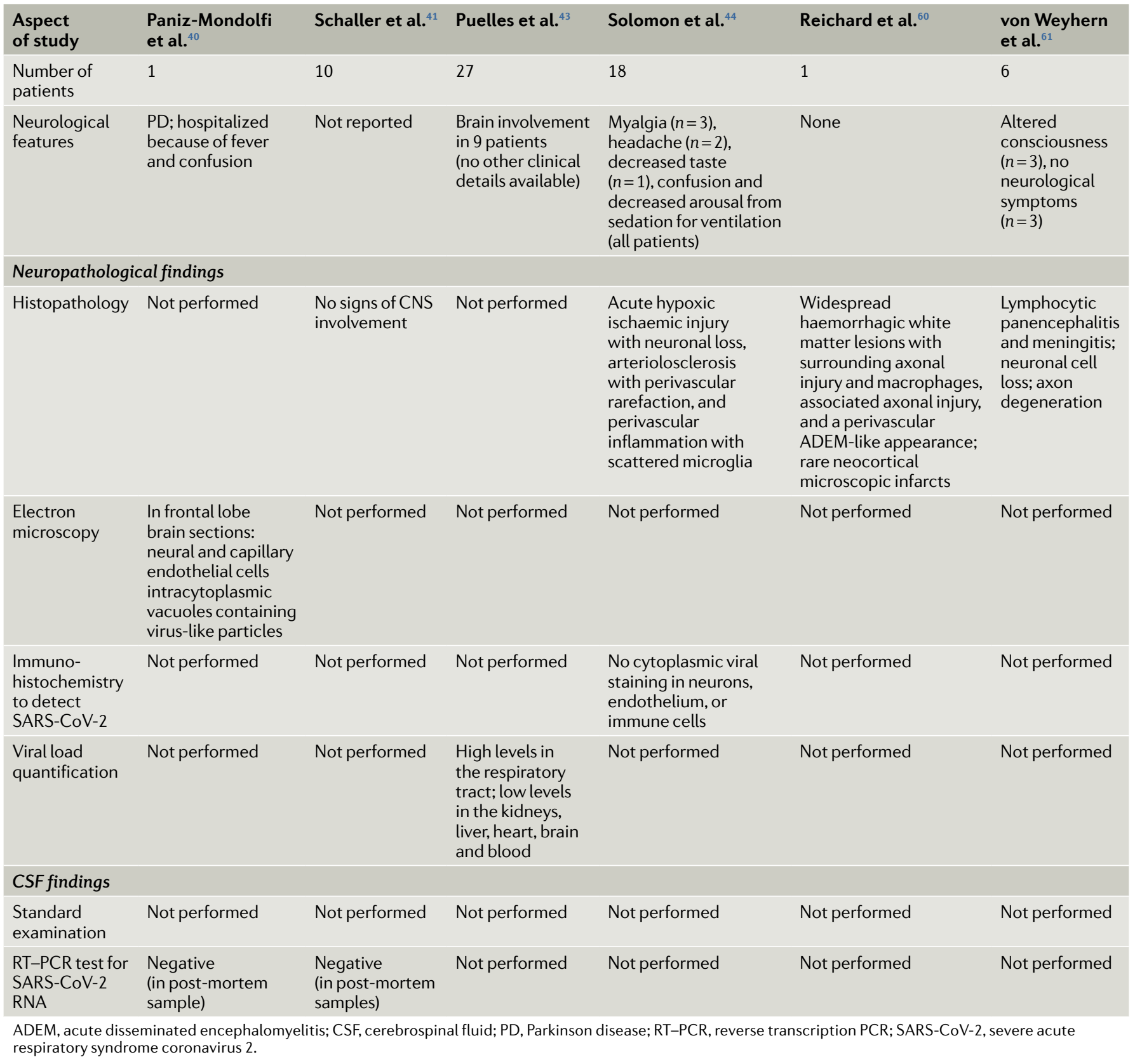

Immune mechanisms. The immunopathogenesis of SARS-CoV-2 is poorly defined at present. Nevertheless, because of the structural homology between SARS-CoV-2 and SARS-CoV, the two viruses might also share similar immunopathogenicity. Experimental evidence indicates that an increased inflammatory response to the virus, characterized by raised levels of cytokines and chemokines, including IL-6, IL-10 and TNF, is likely to have a key role in the early phases of the disease ${ }^{65}$. This response is known as a 'cytokine storm', and severe respiratory manifestations of SARS-CoV-2 infection, such as ARDS, might be a consequence of such a hyperinflammatory state, similar to what is thought to occur in the Kawasaki-like disease that has been observed in children during the COVID-19 outbreak ${ }^{66-68}$. Total serum $\mathrm{T}$ cell concentrations, as well as levels of $\mathrm{CD}^{+}$and $\mathrm{CD}^{+} \mathrm{T}$ cells, show an inverse correlation with cytokine concentration, and are dramatically reduced during the acute phase of the infection, before increasing during disease resolution ${ }^{65}$. Some researchers have suggested that this immune dysregulation, characterized by $\mathrm{T}$ cell depletion and a cytokine storm, might be a bigger contributor to lung tissue injury than the direct damaging effect of the virus, and that such a mechanism might also be operant in the $\mathrm{CNS}^{69,70}$, where it could induce some clinical features, including encephalopathy. On the basis of this hypothesis, it seems possible that immunomodulatory therapies could have a role in the management of many clinical manifestations of COVID-19, including neurological disease.

\section{Specific manifestations}

Taking into account all of the biological pathways summarized above, we consider that some of the most frequent neurological manifestations observed in patients with COVID-19 should be further investigated in 
order to better understand the mechanisms underlying the link between SARS-CoV-2 and the nervous system.

Impaired consciousness. The pathophysiology of impairment of consciousness in patients with COVID-19 is likely to be multifactorial, including direct infection and damage to the parenchyma, as well as toxic-metabolic encephalopathy. Encephalopathy occurs more frequently in patients with severe disease, associated comorbidities, evidence of multiorgan system dysfunction (including hypoxaemia, and renal and hepatic impairment), and elevated markers of systemic inflammation ${ }^{7,9}$. Whether or not encephalitis is a possible manifestation of COVID-19 is a matter of ongoing debate; however, the results of a study of SARS-CoV in a mouse model indicated that spread of the virus within the CNS was associated with relatively limited inflammation ${ }^{71}$. This observation suggests that signs of neuroinflammation could be modest or absent, even in patients with CNS invasion by SARS-CoV-2. At present, only two patients with COVID-19 who fulfil established criteria for the diagnosis of brain inflammation have been reported ${ }^{34,72}$.

PNS disease. Anosmia and dysgeusia are the most frequently reported symptoms resulting from involvement of the PNS in patients with COVID-19 (REF. ${ }^{52}$ ), and these manifestations are considered to be specific to the early stages of infection and useful diagnostic markers ${ }^{51}$ The reported prevalence of anosmia and dysgeusia varies widely, from $~ 5 \%$ among patients hospitalized in Wuhan, China ${ }^{7}$, to approximately $88 \%$ in a multicentre study conducted in Europe ${ }^{73}$. Preliminary reports $^{74,75}$ suggest that olfactory sensory neurons do not express ACE2, thus preventing SARS-CoV-2 from accessing these cells. Conversely, cells in the olfactory epithelium do express ACE2 and are, therefore, vulnerable to SARS-CoV-2 infection. These findings suggest that damage to the olfactory epithelium, rather than neuronal injury, underlies clinical anosmia, which seems to contradict the hypothesis of a direct invasion of the CNS by the virus.

Regarding other neuropathies, although reports are emerging of Guillain-Barré syndrome (GBS) and its variants occurring in patients with COVID-19, the sparse cases described thus far prevent any reliable estimate of incidence. However,
GBS was found in five patients among 1,000-1,200 patients with COVID-19 seen over a 1-month period by Toscano et al. in Northern Italy ${ }^{76}$, and GBS was found in one patient among the 841 patients with COVID-19 included in the ALBACOVID registry ${ }^{8}$. These observations suggest that the incidence of GBS in patients with COVID-19 is much higher than the expected incidence in the general population, which is $\sim 1$ per 100,000 person-years ${ }^{77}$. This finding provides indirect support to the hypothesis that SARS-CoV-2 might induce immune-mediated damage to the nerves after a latent period following the infectious illness. Recent reports of likely immune-mediated conditions, such as $\mathrm{ANE}^{78,79}$ and $\mathrm{ADEM}^{80,81}$, in patients with COVID-19 are in line with this view. Importantly, some patients with SARS-CoV-2-associated GBS do not have any COVID-19 symptoms at presentation ${ }^{82}$. Therefore, clinicians should be aware that GBS might be the first manifestation of SARS-CoV-2 infection in some individuals and should consider signs such as lymphocytopenia and/or thrombocytopenia reasons to suspect COVID-19 in otherwise asymptomatic patients with acute neurological symptoms ${ }^{82}$.
Stroke. Although the exact prevalence of stroke during the outbreak has been difficult to establish, the condition, especially acute brain ischaemia, is emerging as a frequent complication of the COVID-19 pandemic ${ }^{83}$. Viral infections are known to activate a coagulation cascade, and complex crosstalk exists between coagulative haemostasis and inflammation ${ }^{84}$. COVID-19 in particular is commonly complicated by 'sepsis-induced coagulopathy', which is characterized by elevated prothrombin time, elevated D-dimer levels and thrombocytopenia, but without hypofibrinogenaemia ${ }^{84}$. This coagulopathy is related to an infection-induced systemic inflammatory response involving endothelial dysfunction and microthrombosis with organ failure and usually no bleeding ${ }^{85}$. Furthermore, disseminated intravascular coagulation is frequently detected in deceased patients with a diagnosis of COVID-19 (REF. ${ }^{86}$ ). Another important piece of evidence that might explain the presence of coagulation disorders in patients with COVID-19 is the increase in thrombus formation under conditions of hypoxia ${ }^{87}$. Preliminary reports suggest that the risk factors and underlying mechanisms of stroke differ between patients with COVID-19 and

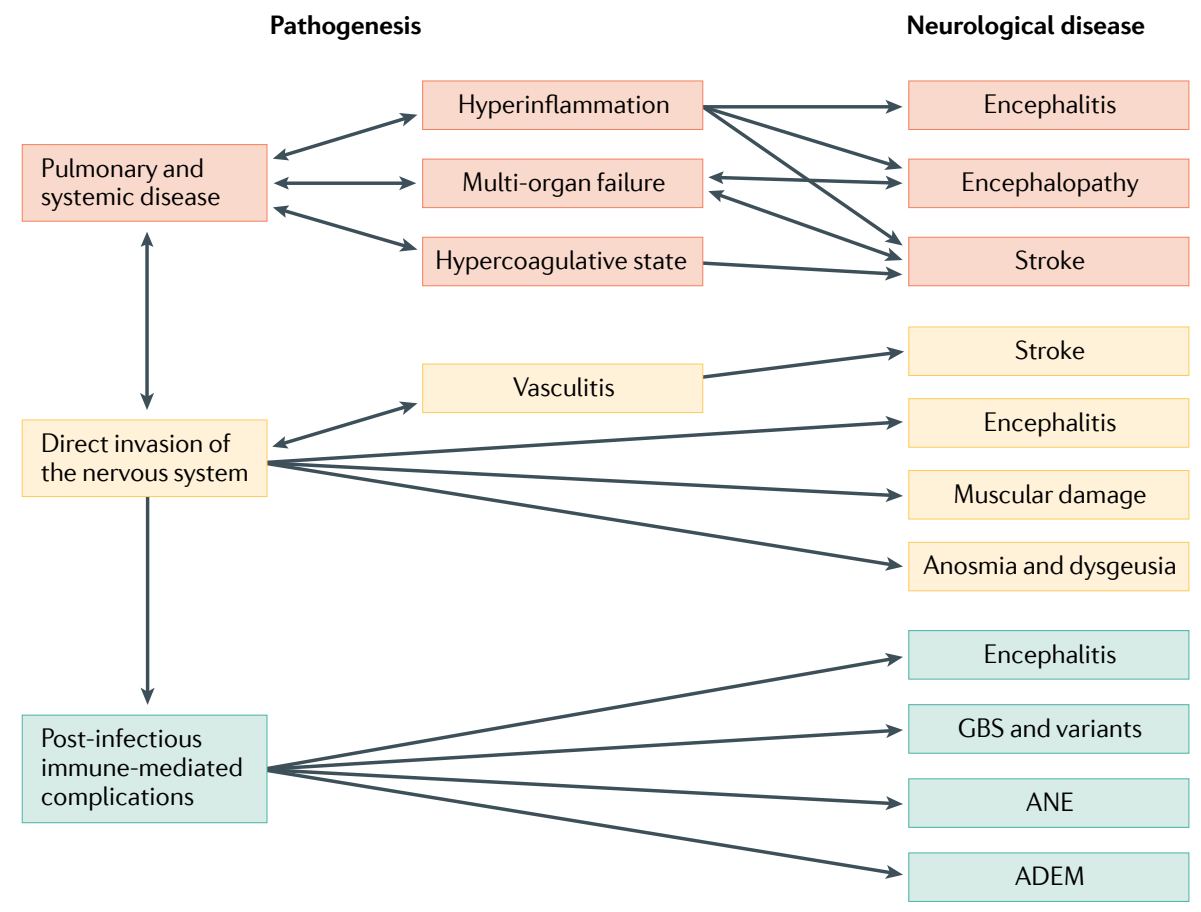

Fig. 1 | Possible mechanisms underlying neurological manifestations in patients with SARS-CoV-2 infection. The neurological diseases that have been observed in individuals with severe acute respiratory syndrome coronavirus 2 (SARS-CoV-2) infection can be split into three categories on the basis of the presumed underlying mechanism. These mechanisms are pulmonary and systemic disease (orange), direct invasion of the nervous system (yellow) and post-infectious immune-mediated complications (green). ADEM, acute disseminated encephalomyelitis; ANE, acute necrotizing encephalopathy; GBS, Guillain-Barré syndrome. 
patients without the infection, with a lower prevalence of hypertension and a higher prevalence of cryptogenic stroke subtype in patients with COVID-19 (REF. ${ }^{88}$ ). Finally, an attractive, yet unproven, hypothesis is that a transient, infection-induced increase in specific pro-coagulant molecules, such as antiphospholipid antibodies, contributes to the risk of brain ischaemia in patients with COVID-19. However, currently, the evidence supporting this hypothesis comes from two small case series of patients with COVID-19 and ischaemic stroke ${ }^{89,90}$ and it should, therefore, be investigated in larger groups.

In addition to inducing hypercoagulable states, SARS-CoV-2 can infect and injure endothelial cells ${ }^{91}$. Evidence indicates that the resulting endothelial dysfunction can lead to systemic arterial and venous microvascular and macrovascular complications $^{92}$, suggesting that similar complications might also occur in the brain. Histological data have highlighted the role of capillary thrombosis in the pathogenesis of organ dysfunction during SARS-CoV-2 infection $^{91}$. Another possibility is that, similar to varicella zoster virus infection ${ }^{93}$, replication of SARS-CoV-2 in the cerebral arterial wall triggers local inflammation, inducing a vasculitic process. However, no evidence of this process occurring in SARS-CoV-2 infection has been reported. Inflammation and apoptosis of endothelial cells after endothelial infection by SARS-CoV-2 has been observed at autopsy in the lung, kidney, bowel and heart $^{94}$, although not yet in cerebral vessels. Inflammatory cells accumulating in the vascular wall might increase blood-brain barrier permeability, activate formation of atherosclerosis in cerebral arteries and contribute to endothelial dysfunction, which, in turn, would affect the automatic regulation of cerebral circulation ${ }^{91}$.

Therefore, COVID-19-induced endothelitis might explain the systemic impaired microcirculatory function observed in different organs in patients with COVID-19 $\left(\mathrm{REF}^{91}{ }^{91}\right.$, contribute to the thrombotic process and increase the risk of brain ischaemia. Whether it is virus-induced injury to endothelial cells (a vasculopathy) or true vasculitis that contributes to COVID-19-related cerebrovascular syndromes remains to be determined.

\section{Conclusions and future directions}

The biological and clinical observations summarized above suggest that SARS-CoV-2 could be responsible for many neurological manifestations, which can be divided into three categories on the basis of the presumed underlying mechanism (FIG. 1). First, neurological consequences of pulmonary disease and associated systemic disease (systemic inflammatory response syndrome, sepsis, and multi-organ failure); this category includes encephalopathy and stroke. Second, neurological manifestations caused by direct invasion of the CNS by the virus; this category could include encephalitis. Last are neurological manifestations caused by post-infectious, immune-mediated complications, including GBS and its variants, ANE and ADEM. As the outbreak continues to spread, our understanding of the neurological manifestations in patients with COVID-19 is also evolving. Nevertheless, more accurate information on COVID-19-associated neurological manifestations is obviously needed. To address this need, studies need to be carefully designed, taking into account several methodological issues.

A key challenge in any epidemiological investigation is the precise definition of patients' clinical phenotypes. Clinicians should be aware that the diagnostic work-up should be as detailed and exhaustive as possible in order to rule out causes other than SARS-CoV-2 infection before including cases in epidemiological analyses. This requires, for example: a distinction between patients with clear evidence of brain inflammation (encephalitis) and patients with encephalopathy; a careful characterization of all patients with suspected disease of the peripheral nerves by CSF examination, neurophysiological studies and, when needed, spinal imaging; and the use of brain digital subtraction angiography or cerebral vessel wall imaging in patients with presumed cerebral vasculitis. Although this careful characterization is not always easy, especially in severely affected individuals, it is noteworthy that such a rigorous diagnostic approach was not applied in many of the studies published to date, with the obvious consequence of phenotypic heterogeneity and, therefore, unreliable findings. A useful experimental approach would be, at the least, a large-scale case-control study to compare homogeneous groups of patients with confirmed SARS-CoV-2 infection with non-infected individuals; however, this approach would present design challenges as exposure to SARS-CoV-2 is high in the general population and widespread antibody testing would be needed to ascertain seroprevalence.

After careful clinical characterization, blood samples and CSF samples should be collected longitudinally to enable the evaluation of systemic and CNS inflammatory markers, which might enable the identification of specific subgroups of COVID-19 patients more prone to developing neurological alterations. In addition, neuropathological examination of patients with COVID-19 after death should be performed, as this approach might provide clues as to the mechanisms underlying nervous system injury. Finally, although emphasis has been put on the recovery from the acute phase of the infection, the potential long-term neurological effects of COVID-19 should not be overlooked. If SARS-CoV-2 invades the CNS, neurological manifestations could reappear in predisposed individuals after the virus has remained latent for a long time. Longitudinal neurological assessments of patients after recovery will be crucial in understanding the natural history of SARS-CoV-2 in the CNS and monitoring for potential neurological sequelae. Evidence from animal and human studies of other

\section{Glossary \\ Acute disseminated encephalomyelitis \\ (ADEM). An immune-mediated inflammatory condition that causes myelin damage and predominantly affects the white matter of the brain and spinal cord. \\ Acute respiratory distress syndrome (ARDS). A type of acute diffuse, inflammatory lung injury that causes respiratory insufficiency. \\ D-dimer \\ A fibrin degradation product, the concentration of which can be determined by a blood test and can help} diagnose thrombosis.

Fluid-attenuated inversion recovery (FLAIR). An MRI sequence based on tissue T2 prolongation that suppresses the cerebrospinal fluid signal interference and minimizes contrast between grey matter and white matter.

Posterior reversible encephalopathy syndrome (PRES). A clinicoradiological syndrome characterized by headache, seizures, altered mental status, visual loss and white matter vasogenic oedema that predominantly affects the posterior occipital and parietal lobes of the brain

Prothrombin time

The time it takes plasma to clot after addition of tissue factor; used as a laboratory test of the clotting tendency of blood.

Real-time reverse transcription PCR

(Real-time RT-PCR). A technique combining reverse transcription of RNA into DNA and amplification of specific DNA targets using PCR; this amplification reaction is monitored using fluorescence to measure the amount of a specific RNA.

Susceptibility-weighted imaging

(SWI). An MRI technique that exploits the magnetic susceptibility differences of various compounds,

such as blood, iron and diamagnetic calcium, for the differential diagnosis of many neurological disorders. 
coronaviruses suggests that, in some at-risk individuals, the inflammatory response elicited in acute or chronic infection might trigger or accelerate subclinical mechanisms that underlie the earliest stages of many neurological diseases, for example, neurodegenerative disorders ${ }^{95-98}$. Ideally, longitudinal studies should include careful neurological, imaging, laboratory and neuropsychological evaluation to examine multiple cognitive domains, in order to determine to what extent the interplay between central and systemic infection drives CNS damage and neurological alterations.

\section{Alessandro Pezzini (iD ${ }^{凶}$ and Alessandro Padovani}

Department of Clinical and Experimental Sciences, Neurology Clinic, University of Brescia, Brescia, Italy.

凶e-mail: alessandro.pezzini@unibs.it

\section{https://doi.org/10.1038/s41582-020-0398-3}

Published online 24 August 2020

1. World Health Organization. Coronavirus disease 2019 (COVID-19) Situation Report-51. https://www.who. int/docs/default-source/coronaviruse/situationreports/20200311-sitrep-51-covid-19.pdf?sfvrsn= 1 ba62e57 10 (WHO, 2020)

2. Zou, L. et al. SARS-CoV-2 viral load in upper respiratory specimens of infected patients. N. Engl. J. Med. 382 1177-1179 (2020)

3. World Health Organization. Report of the WHO China Joint Mission on Coronavirus Disease 2019 (COVID-19). https://www.who.int/publications/i/item/ report-of-the-who-china-joint-mission-on-coronavirusdisease-2019-(covid-19) (WHO, 2020).

4. Zhang, H., Penninger, J. M., Li, Y., Zhong, N. \& Slutsky, A. S. Angiotensin-converting enzyme 2 (ACE2) as a SARS-CoV-2 receptor: molecular mechanisms and potential therapeutic target. Intensive Care Med. 46, 586-590 (2020)

5. Zubair, A. S. et al. Neuropathogenesis and neurologic manifestations of the coronaviruses in the age of coronavirus disease 2019: a review. JAMA Neurol. https://doi.org/10.1001/jamaneurol.2020.2065 (2020).

6. Koralnik, I. J. \& Tyler, K L. COVID-19: a global threat to the nervous system. Ann. Neurol 88, 1-11 (2020)

7. Mao, L. et al. Neurologic manifestations of hospitalized patients with coronavirus disease 2019 in Wuhan, China. JAMA Neurol. 77, 1-9 (2020).

8. Romero-Sánchez, C. M. et al. Neurologic manifestations in hospitalized patients with COVID-19: the ALBACOVID registry. Neurology https://doi.org/ 10.1212/WNL.0000000000009937 (2020).

9. Pinna, P. et al. Neurological manifestations and COVID-19: experiences from a tertiary care center at the frontline. J. Neurol. Sci. 415, 116969 (2020).

10. Karadaş, Ö., Öztürk, B. ¿ Sonkaya, A. R. A prospective clinical study of detailed neurological manifestations in patients with COVID-19. Neurol. Sci. 41, 1991-1995 (2020).

11. Xiong, W. et al. New onset neurologic events in people with COVID-19 infection in three regions in China. Neurology https://doi.org/10.1212/WNL. 0000000000010034 (2020).

12. Helms, J. et al. Neurologic features in severe SARSCoV-2 infection. N. Engl. J. Med. 382, 2268-2270 (2020).

13. Benussi, A. et al. Clinical characteristics and outcomes of inpatients with neurologic disease and COVID-19 in Brescia, Lombardy, Italy. Neurology https://doi.org/ 10.1212/WNL.0000000000009848 (2020).

14. Paterson, R. W. et al. The emerging spectrum of COVID-19 neurology: clinical, radiological and laboratory findings. Brain https://doi.org/10.1093/ brain/awaa240 (2020).

15. Chen, T. et al. Clinical characteristics of 113 deceased patients with coronavirus disease 2019: retrospective study. BMJ 368, m 1091 (2020).

16. Chan, J. W. M. et al. Short term outcome and risk factors for adverse clinical outcomes in adults with severe acute respiratory syndrome (SARS). Thorax $\mathbf{5 8}$ 686-689 (2003).

17. Badawi, A. \& Ryoo, S. G. Prevalence of comorbidities in the Middle East respiratory syndrome coronavirus (MERS-CoV): a systematic review and meta-analysis. Int. J. Infect. Dis. 49, 129-133 (2016).

18. Andersen, K. G., Rambaut, A., Lipkin, W. I. Holmes, E. C. \& Garry, R. F. The proximal origin of SARS-CoV-2. Nat. Med. 4, 450-452 (2020).

19. Zhang, T. W. Q. \& Zhang, Z. Probable pangolin origin of SARS-CoV-2 associated with the COVID-19 outbreak. Curr. Biol. 7, 1346-1351 (2020).

20. Arbour, N. et al. Acute and persistent infection of human neural cell lines by human coronavirus OC43. J. Virol. 73, 3338-3350 (1999).

21. Arbour, N., Day, R., Newcombe, J. \& Talbot, P. J. Neuroinvasion by human respiratory coronaviruses. J. Virol. 74, 8913-8921 (2000)

22. Arbour, N. et al. Persistent infection of human oligodendrocytic and neuroglial cell lines by human coronavirus 229E. J. Virol. 73, 3326-3337 (1999).

23. Bonavia, A., Arbour N., Yong, V. W. \& Talbot, P. J. Infection of primary cultures of human neural cells by human coronaviruses 229E and OC43. J. Virol. 71, 800-806 (1997).

24. $\mathrm{Gu}$, J. et al. Multiple organ infection and the pathogenesis of SARS. J. Exp. Med. 202, 415-424 (2005).

25. Xu, J. et al. Detection of severe acute respiratory syndrome coronavirus in the brain: potential role of the chemokine Mig in pathogenesis. Clin. Infect. Dis. 41, 1089-1096 (2005)

26. Zhou, P. et al. A pneumonia outbreak associated with a new coronavirus of probable bat origin. Nature 579 270-273 (2020)

27. Baig, A. M., Khaleeq, A., Ali, U. \& Syeda, H. Evidence of the COVID-19 virus targeting the CNS: tissue distribution, host-virus interaction, and proposed neurotropic mechanisms. ACS Chem. Neurosci. 11, 995-998 (2020).

28. Brian, D. A. \& Baric, R. S. Coronavirus genome structure and replication. Curr. Top. Microbiol. Immunol. 287, 1-30 (2005).

29. Greig, A. S. et al. A hemagglutinating virus producing encephalomyelitis in baby pigs. Can. J. Comp. Med. vet. Sci. 26, 49-56 (1962).

30. Foley, J. E., Rand, C. \& Leutenegger, C. Inflammation and changes in cytokine levels in neurological feline infectious peritonitis. J. Feline Med. Surg. 5, 313-322 (2003).

31. Lampert, P. W., Sims, J. K. \& Kniazeff, A. J. Mechanism of demyelination in JHM virus encephalomyelitis. Electron microscopic studies. Acta Neuropathol. 24 76-85 (1973)

32. Hosking, M. P. \& Lane, T. E. The pathogenesis of murine coronavirus infection of the central nervous system. Crit. Rev. Immunol. 30, 119-130 (2010).

33. Weiss, S. R. \& Leibowitz, J. L. in Advances in Virus Research Vol. 81 (eds Maramorosch, K., Shatkin, A. J. ¿ Murphy, F. A.) 85-164 (Elsevier, 2011).

34. Moriguchi, T. et al. A first case of meningitis/ encephalitis associated with SARS Coronavirus-2. Int. J. Infect. Dis. 94, 55-58 (2020).

35. Domingues, R. B. et al. First case of SARS-COV-2 sequencing in cerebrospinal fluid of a patient with suspected demyelinating disease. $J$ Neurol. https:// doi.org/10.1007/s00415-020-09996-w (2020).

36. Huanga, Y. H., Jianga, D. \& Huangb, J. T. SARS-CoV-2 detected in cerebrospinal fluid by PCR in a case of COVID-19 encephalitis. Brain Behav. Immun. 87, 149 (2020).

37. Virhammar, J. et al. Acute necrotizing encephalopathy with SARS-CoV-2 RNA confirmed in cerebrospinal fluid. Neurology https://doi.org/10.1212/WNL. 0000000000010250 (2020)

38. Kremer, S. et al. Brain MRI findings in severe COVID-19: a retrospective observational study. Radiology https:// doi.org/10.1148/radiol.2020202222 (2020).

39. Filatov, A., Sharma, P., Hindi, F. \& Espinosa, P. S. Neurological complications of coronavirus disease (COVID-19): encephalopathy. Cureus 12, e7352 (2020).

40. Paniz-Mondolfi, A. et al. Central nervous system involvement by severe acute respiratory syndrome coronavirus-2 (SARS-CoV-2). J. Med. Virol. 92, 699-702 (2020)

41. Schaller, T. et al. Postmortem examination of patients with COVID-19. JAMA 323, 2518-2520 (2020).

42. DeBiasi, R. L. \& Tyler, K. L. Polymerase chain reaction in the diagnosis and management of central nervous system infections. Arch. Neurol. 10, 1215-1219 (1999).
43. Puelles, V. G. et al. Multiorgan and renal tropism of SARS-CoV-2. N. Engl. J. Med. https://doi.org/10.1056/ NEJMc2011400 (2020).

44. Solomon, I. H. et al. Neuropathological features of Covid-19. N. Engl. J. Med. https://doi.org/10.1056/ NEJMc2019373 (2020).

45. Berth, S. H., Leopold, P. L. \& Morfini, G. N. Virusinduced neuronal dysfunction and degeneration. Front. Biosci. 14, 5239-5259 (2009).

46. Desforges, M., Le Coupanec, A., Brison, E. Meessen-Pinard, M. \& Talbot, P. J. Neuroinvasive and neurotropic human respiratory coronaviruses: potential neurovirulent agents in humans. Adv. Exp. Med. Biol. 807, 75-96 (2014).

47. Desforges, M. et al. Human coronaviruses and other respiratory viruses: underestimated opportunistic pathogens of the central nervous system? Viruses 12 14 (2019).

48. Talbot, P. J., Jacomy, H. \& Desforges, M. in Nidoviruses (eds Perlman, S., Gallagher, T. \& Snijder, E. J.) 313-324 (ASM Press, 2008)

49. Li, Y C., Bai, W. Z Hirano, N., Hayashida, T \& Hashikawa, T. Coronavirus infection of rat dorsal root ganglia: ultrastructural characterization of viral replication, transfer, and the early response of satellite cells. Virus Res. 163, 628-635 (2012).

50. van Riel, D., Verdijk, R. \& Kuiken, T. The olfactory nerve: a shortcut for influenza and other viral diseases into the central nervous system. J. Pathol. 235 277-287 (2015)

51. Cooper, K. W. et al. COVID-19 and the chemical senses: supporting players take center stage. Neuron 107, 219-233 (2020)

52. $\mathrm{Li}, \mathrm{W}$. et al. Angiotensin-converting enzyme 2 is a functional receptor for the SARS coronavirus. Nature 426, 450-454 (2003).

53. Tan, H. W., Xu, Y. M. \& Lau, A. T. Y. Angiotensinconverting enzyme 2 : the old door for new severe acute respiratory syndrome coronavirus 2 infection. Rev. Med. Virol. https://doi.org/10.1002/rmv.2122 (2020).

54. Shang, J. et al. Structural basis of receptor recognition by SARS-CoV-2. Nature 581, 221-224 (2020).

55. Hoffmann, M. et al. SARS-CoV-2 cell entry depends on ACE2 and TMPRSS2 and is blocked by a clinically proven protease inhibitor. Cell 181, 271-280.e8 (2020).

56. Simmons, G. et al. Inhibitors of cathepsin L prevent severe acute respiratory syndrome coronavirus entry. Proc. Natl Acad. Sci. USA 102, 11876-11881 (2005).

57. Iwata-Yoshikawa, N. et al. TMPRSS2 contributes to virus spread and immunopathology in the airways of murine models after coronavirus infection. J. Virol. 93 , e01815-e01818 (2019).

58. Zhou, Y. et al. Protease inhibitors targeting coronavirus and filovirus entry. Antivir. Res. 116, 76-84 (2015).

59. Chen, R. et al. The spatial and cell-type distribution of SARS-CoV-2 receptor ACE2 in human and mouse brain. Preprint at bioRxiv https://doi.org/10.1101/202 0.04.07.030650v1 (2020).

60. Reichard, R. R. et al. Neuropathology of COVID-19 a spectrum of vascular and acute disseminated encephalomyelitis (ADEM)-like pathology. Acta Neuropathol. 140, 1-6 (2020).

61. von Weyhern, C. H., Kaufmann, I., Neff, F. \& Kremer, M Early evidence of pronounced brain involvement in fatal COVID-19 outcomes. Lancet 395, e 109 (2020).

62. Nicholson, P., Alshafai, L. \& Krings, T. Neuroimaging findings in patients with COVID-19. AJNR Am.J. Neuroradiol. https://doi.org/10.3174/ajnr.A6630 (2020).

63. Coolen, T. et al. Early postmortem brain MRI findings in COVID-19 non-survivors. Neurology https://doi.org/ 10.1212/WNL.0000000000010116 (2020).

64. Radmanesh, A. et al. COVID-19-associated diffuse leukoencephalopathy and microhemorrhages. Radiology https://doi.org/10.1148/radiol.2020202040 (2020).

65. Diao, B. et al. Reduction and functional exhaustion of T cells in patients with coronavirus disease 2019 (COVID-19). Front. Immunol. 11, 827 (2020).

66. Bryce, C. et al. Pathophysiology of SARS-CoV-2 targeting of endothelial cells renders a complex disease with thrombotic microangiopathy and aberrant immune response. The Mount Sinai COVID-19 autopsy experience. Preprint at medRxiv https://doi.org/10.1101/2020.05.18.20099960v1 (2020).

67. Licciardi, F. et al. SARS-CoV-2-induced Kawasaki-like hyperinflammatory syndrome: a novel COVID phenotype in children. Pediatrics 146, e20201711 (2020). 
68. Toubiana, J. et al. Kawasaki-like multisystem inflammatory syndrome in children during the covid-19 pandemic in Paris, France: prospective observational study. BMJ 369, m2094 (2020)

69. Pedersen, S. F. \& Ho, Y. C. SARS-CoV-2: a storm is raging. J. Clin. Invest. 130, 2202-2205 (2020).

70. Li, C. K. et al. T cell responses to whole SARS coronavirus in humans. J. Immunol. 181, 5490-5500 (2008).

71. Netland, J. et al. Severe acute respiratory syndrome coronavirus infection causes neuronal death in the absence of encephalitis in mice transgenic for human ACE2. J. Virol. 82, 7264-7275 (2008).

72. Pilotto, A. et al. Steroid-responsive encephalitis in Coronavirus Disease 2019. Ann. Neurol. https://doi.org/ 10.1002/ana.25783 (2020).

73. Lechien, J. R et al. Olfactory and gustatory dysfunctions as a clinical presentation of mildto-moderate forms of the coronavirus disease (COVID-19): a multicenter European study. Eur. Arch Otorhinolaryngol. 277, 2251-2261 (2020).

74. Brann, D., Tsukahara, T., Weinreb, C., Logan, D. W. \& Datta, S. R. Non-neural expression of SARS-CoV-2 entry genes in the olfactory epithelium suggests mechanisms underlying anosmia in COVID-19 patients. Pre-print at bioRxiv https://doi.org/10.1101/2020.03. 25.009084v4 (2020)

75. Fodoulian, L. et al. SARS-CoV-2 receptor and entry genes are expressed by sustentacular cells in the human olfactory neuroepithelium. Pre-print at bioRxiv https://doi.org/10.1101/2020.03.31.013268v2 (2020).

76. Toscano, G. et al. Guillain-Barré syndrome associated with SARS-CoV-2. N. Engl. J. 382, 2574-2576 (2020).

77. Willison, H. J., Jacobs, B. C. \& van Doorn, P. A. Guillain-Barrê syndrome. Lancet 388, 717-727 (2016).

78. Poyiadji, N. et al. COVID-19-associated acute hemorrhagic necrotizing encephalopathy: imaging features. Radiology 296, E119-E120 (2020).

79. Dixon, L. et al. COVID-19-related acute necrotizing encephalopathy with brain stem involvement in a patient with aplastic anemia. Neurol. Neuroimmunol. Neuroinflamm 7, e789 (2020).
80. Zhang, T., Rodricks, M. B. \& Hirsh, E. COVID-19associated acute disseminated encephalomyelitis: a case report. Pre-print at medRxiv. https://doi.org/ 10.1101/2020.04.16.20068148v1 (2020).

81. Zhao, K. et al. Acute myelitis after SARS-CoV-2 infection: a case report. Pre-print at medRxiv. https://doi.org/ $10.1101 / 2020.03 .16 .20035105 \mathrm{v} 2$ (2020).

82. Dalakas, M. C. Guillain-Barré syndrome: the first documented COVID-19-triggered autoimmune neurologic disease. More to come with myositis in the offing. Neurol. Neuroimmunol. Neuroinflamm. 7, e781 (2020).

83. Zhao, J., Rudd, A. \& Liu, R. Challenges and potential solutions of stroke care during the coronavirus disease 2019 (COVID-19) outbreak. Stroke 51, 1356-1357 (2020).

84. Al-Ania, F Chehadea, S. \& Lazo-Langnera, A Thrombosis risk associated with COVID-19 infection. A scoping review. Thromb. Res. 192, 152-160 (2020).

85. Iba, T. et al. Diagnosis and management of sepsisinduced coagulopathy and disseminated intravascular coagulation. J. Thromb. Haemost. 17, 1989-1994 (2019).

86. Wichmann, D. et al. Autopsy findings and venous thromboembolism in patients with COVID-19: a prospective cohort study. Ann. Intern. Med. https://doi.org/10.7326/M20-2003 (2020).

87. Gupta, N. et al. The stimulation of thrombosis by hypoxia. Thromb. Res. 181, 77-83 (2019)

88. Yaghi, S. et al. SARS-CoV-2 and stroke in a New York healthcare system. Stroke 51, 2002-2011 (2020).

89. Beyrouti, R. et al. Characteristics of ischaemic stroke associated with COVID-19. J. Neurol. Neurosurg. Psychiatry 91, 889-891 (2020)

90. Zhang, Y. et al. Coagulopathy and antiphospholipid antibodies in patients with Covid-19. N. Engl. J. Med. 382, e38 (2020).

91. Pons, S., Fodil, S., Azoulay, E. \& Zafrani, L. The vascular endothelium: the cornerstone of organ dysfunction in severe SARS-CoV-2 infection. Crit. Care 24, 353 (2020).

92. Klok, F A et al. Incidence of thrombotic complications in critically ill ICU patients with COVID-19. Thromb. Res. 191, 145-147 (2020).
93. Gilden, D., Cohrs, R. J., Mahalingam, R. \& Nagel, M. A. Varicella zoster virus vasculopathies: diverse clinical manifestations, laboratory features, pathogenesis, and treatment. Lancet Neurol. 8, 731-740 (2009).

94. Varga, Z. et al. Endothelial cell infection and endotheliitis in COVID-19. Lancet 395, 1417-1418 (2020).

95. Fazzini, E., Fleming, J. \& Fahn, S. Cerebrospinal fluid antibodies to coronavirus in patients with Parkinson's disease. Mov. Disord. 7, 153-158 (1992)

96. Ann Yeh, E., Collins, A., Cohen, M. E., Duffner, P. K. $\&$ Faden, $H$. Detection of coronavirus in the central nervous system of a child with acute disseminated encephalomyelitis. Pediatrics 113, e73-e76 (2004).

97. Dessau, R. B., Lisby, G. \& Frederiksen, J. L.

Coronaviruses in brain tissue from patients with multiple sclerosis. Acta Neuropathol. 101, 601-604 (2001).

98. Savarin, C. \& Bergmann, C. C. Viral-induced suppression of self-reactive $T$ cells: lessons from neurotropic coronavirus-induced demyelination. J. Neuroimmunol. 308, 12-16 (2017).

\section{Author contributions}

A. Pezzini researched data for the article, made a substantial contribution to the discussion of content, wrote the article, and reviewed and edited the manuscript before submission. A. Padovani made a substantial contribution to discussion of content, and reviewed and edited the manuscript before submission.

\section{Competing interests}

The authors declare no competing interests.

\section{Peer review information}

Nature Reviews Neurology thanks C. Bergmann, J. Frontera and the other, anonymous, reviewer(s) for their contribution to the peer review of this work.

\section{Publisher's note}

Springer Nature remains neutral with regard to jurisdictional claims in published maps and institutional affiliations.

(c) Springer Nature Limited 2020 